\title{
Growth cone guidance in the zebrafish central nervous system
}

\author{
John Y. Kuwada \\ University of Michigan, Ann Arbor, Michigan, USA
}

\begin{abstract}
The accessibility and simplicity of the zebrafish embryo have allowed researchers to make a detailed characterization of pathfinding by identifiable growth cones. The growth cones follow precise cell-specific pathways to their targets. Analyses of pathfinding in mutant and experimentally manipulated wild type embryos have shown that growth cones accomplish this by interacting with specific cellular cues in their environment, many of which are likely to be redundant.
\end{abstract}

Current Opinion in Neurobiology 1992, 2:31-35

\section{Introduction}

The zebrafish embryo is quickly being established as an excellent vertebrate embryo for studying the processes of development with a combination of genetic, molecular, and cellular techniques. One problem that is being fruitfully addressed is how growth cones navigate through the embryos to find their targets. The clarity and simplicity of the early embryo, combined with the availability of mutations and the ability to ablate and transplant specific cells, have been particularly useful in elucidating the mechanisms that direct growth cones along their pathways. This review focuses on the recent analyses of pathfinding by growth cones in the central nervous system of early zebrafish embryos, but also includes recent, relevant studies in other vertebrate and invertebrate embryos. Aspects of pathfinding by zebrafish motor neurons are discussed in another review (M Westerfield, this issue, pp 28-30).

\section{Pathfinding is precise and cell-specific}

The central nervous system of the early zebrafish embryo contains a simple scaffold of axonal tracts that are pioneered by the growth cones of specific classes of neurons in the spinal cord $\left[1,2,3^{\circ}\right]$ and small clusters of neurons in the brain $[4 \bullet, 5 \bullet, 6]$. These early growth cones follow precise and cell-specific pathways to establish this scaffold of tracts. In some cases, for instance for some classes of spinal interneurons, pioneer growth cones interact with a specific set of cells that act as intermediate targets (see below). In other cases, such as the epiphysial neurons in the zebrafish brain and Rohon-Beard neurons in the medaka fish spinal cord, pioneer growth cones extend along the endfeet of neuroepithelial cells found on the superficial surface of the central nervous system, and do not appear to encounter any organized set of channels or other cellular features that could potentially guide them $[6,7]$. This opens up the possibility that spatially restricted subsets of the endfeet of neuroepithelial cells may express molecules which promote or direct axonal outgrowth differentially. In fact, the endfeet of neuroepithelial cells may serve as part of an ensemble of redundant pathfinding cues for some follower growth cones in the zebrafish brain (see below).

Growth cones that are projected later in development also follow stereotyped cell-specific routes within the early scaffold of axons in the zebrafish embryo [4*]. The stereotyped pattern of axonal outgrowth seen in zebrafish embryos is similar to that found in the embryos of a wide variety of animals including insects [8] and mammals [9*].

\section{Extrinsic cues guide growth cones}

\section{Spinal cord}

The detailed description of pathfinding by identified growth cones in the zebrafish embryo has indicated sites where growth cones may potentially select their correct pathway from a number of other pathways, and has helped to determine potential sources of guidance information. In the spinal cord, one such site is the floor plate. The floor plate has been identified as a site where growth cones make very precise turns in the rat spinal cord [10*] as well as in other animals $[11,12]$. This site has also been identified as the area where the axons of rat commissural neurons switch from expressing one axonal adhesion molecule to another [13]. In the zebrafish spinal cord, the growth cones of two classes of interneurons (CoPA and VeLD) exhibit cell-specific turns near the ventral midline of the cord in the floor plate region (Fig. 1a)

\footnotetext{
Abbreviations

MLF-medial longitudinal fasciculus; nucPC — nucleus of the posterior commissure; PC—posterior commissure; TPOC - tract of the postoptic commissure.
} 
[3•]. Both the COPA and VeLD neurons initially project growth cones toward the ventral midline, but once near the midline their growth cones diverge. The VeLD growth cone turns posteriorly without crossing the midline to pioneer a ventrolateral longitudinal tract, while the CoPA growth cone crosses the midline and turns anteriorly, eventually ascending in a dorsolateral longitudinal tract. Not only is the floor plate the site of the turns made by the growth cones, but it also emits a chemotropic signal which attracts the growth cones of commissural neurons in both mammals [14] and chicks [15.] (see the review by $M$ Tessier-Iavigne, this issue, pp 60-65). In light of these findings, it has been proposed that the floor plate serves as an intermediate target which provides multiple cell-specific guidance cues in the zebrafish spinal cord.

(a)

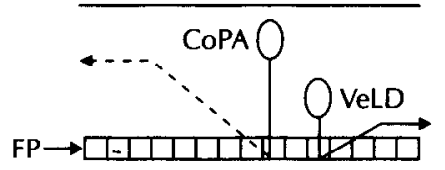

(b)

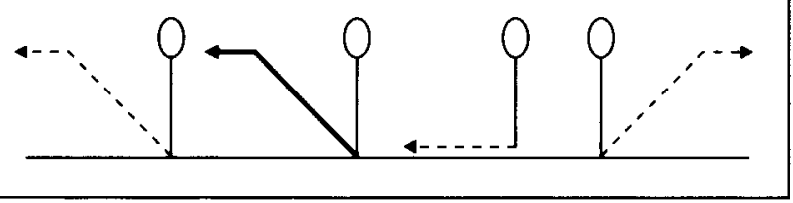

Fig. 1. (a) A schematic diagram showing the relationship of the COPA and VeLD axons to the floor plate (FP) in a sideview of the early zebrafish spinal cord. Dashed lines indicate axons which have crossed the ventral midline. Anterior is to the left. (b) Four examples of the axonal trajectories of CoPA neurons in cyc-1 embryos and in wildtype embryos following laser ablation of the midline floor plate cells. CoPA growth cones followed either normal or aberrant pathways.

This hypothesis has recently been tested by examination of pathfinding by the VeLD and CoPA growth cones in the absence of a morphologically and antigenically distinct subset of floor plate cells, the midline floor plate cells (RR Bernhardt and JY Kuwada: Soc Neurosci Abstr 1990, 16:309; JY Kuwada and K Hatta: Soc Neurosci Abstr 1990, 16:309). These cells form a single row which straddles the ventral midline. The midline floor plate cells were specifically eliminated either by laser ablation prior to axonal outgrowth or by the $c y c-1$ mutation. The $c y c-1(b 16)$ mutation results in a pleiotropic recessive phenotype that is $92 \%$ penetrant [ $\left.160^{\circ}\right]$. In the mutant cord the phenotype appears to be limited to the deletion of the midline floor plate cells, which never appear to develop. In both mutant and laser ablated embryos, the absence of the midline floor plate cells did not prevent outgrowth towards the ventral midline by the VeLD and CoPA growth cones. Once near the midline, however, some growth cones followed aberrant pathways while others followed correct pathways (Fig. 1b). For example, CoPA growth cones followed aberrant pathways in $57 \%$ of cases in cyc-1 embryos, compared to $1 \%$ in wildtype embryos. The range of aberrant pathways taken by the CoPA and
VeLD growth cones were similar in both $c y c$ - 1 cmbryos and embryos after laser ablation of the midline floor plate cells. For example, CoPA growth cones followed an ipsilateral ascending pathway, a ventral ascending pathway, and a descending pathway, in both $c y c-1$ and laser-ab. lated embryos as opposed to the normal contralateral ascending pathway. These effects on growth cones were cell-specific, other classes of spinal neurons were not affected. The findings demonstrate that the midline floor plate cells are not necessary for outgrowth by growth cones towards the ventral midline, but are necessary for normal error-free pathfinding at the midline. Thus, the midline floor plate cells appear to function as intermediate targets that provide multiple cell-specific guidance cues.

The floor plate cells may also serve as an intermediate target for reticulospinal axons in the zebrafish hindbrain. Many reticulospinal neurons project an axon along a tract which is located just lateral to the floor plate region [17]. These axons are also disarrayed in the hindbrain of $c y c$ 1 and floor plate ablated embryos (K Hatta et al: Soc Neurosci Abstr 1990, 16:310).

The flip side of the experiments in the spinal cord is that a significant number of both CoPA and VeLD growth cones followed their normal pathways despite the absence of the midline floor plate cells. The floor plate region usually consists of three neuroepithelial cells in cross-section, one on either side of the midline cell [3•] (see [16*•] for an alternative interpretation). Thus, the variable behavior of growth cones in the absence of the midline cell may be due to a quantitative decrease in a cue normally provided by the entire floor plate region. This, however, appears not to be the case. First, ablation of one of the cells lateral to the midline floor plate cells induces no errors by CoPA growth cones (RR Bernhardt, $\mathrm{N}$ Nguyen and JY Kuwada, unpublished data). Second, ablation of all the floor plate cells (the midline and lateral cells) in wildtype embryos, and neuroepithelial cells near the ventral midline in cyc- 1 embryos, did not prevent CoPA growth cones from extending to the midline along their normal route. Once at the midline their error rate was not significantly higher than that seen in unmanipulated $c y c-1$ embryos (RR Bernhardt and JY Kuwada, unpublished data).

The variable trajectories followed in the absence of the midline floor plate cells may indicate that a second source of pathfinding cues exist near the ventral midline. Two likely candidates are the basal lamina under the floor plate and the notochord. CoPA growth cones cross the midline by extending between the ventral surface of the floor plate cells and the basal lamina. The notochord is located just ventral to the spinal cord and contacts the cord during the entire period growth cones are approaching and crossing the midline [3*]. If guidance cues are deposited on the basal lamina, then the source of the secreted cue could either be the floor plate cells or the notochord. Of the two cell types, the notochord is more likely to be a source as in cyc-1 embryos the midline floor plate cells never appear to develop [16*0].

If the notochord is another source of pathfinding cues, then elimination of the notochord would be expected 
to induce errors in pathfinding, and elimination of both the notochord and floor plate would perhaps induce a higher rate of crrors than would the elimination of the floor plate or notochord alone. In fact, disruption of the normal pattern of axons does occur in Xenopus and mouse embryos when the notochord and floor plate are missing $[18,19]$. Ultraviolet irradiation of the vegetal poles of Xenopus embryos prior to the first cleavage produces embryos which lack both the notochord and floor plate. Likewise, in the caudal regions of the neuraxis of embryos afflicted with the Danforth's short tail $(S d)$ mutation, the spinal cord is missing both the notochord and floor plate. In both cases some spinal axons are disarrayed, but it is not presently possible to ascribe these defects to either the missing floor plate or the notochord, or both, as they were both absent.

In the anterior region of the neuraxis of the $S d$ embryos the spinal cords are sometimes missing the notochord but not the floor plate. In these embryos the pattern of commissural axons in cross sections of rostral regions of the spinal cord appear identical to those taken from sibling wildtype embryos. This demonstrates that the initial circumferential portions of commissural axons to the floor plate were not obviously perturbed. The trajectories of these axons once at the floor plate, however, are difficult to discern from cross sections of spinal cords. For example, mistakes such as turning ipsilaterally rather than contralaterally, or posteriorly rather than anteriorly, in the floor plate region by some but not all axons would be difficult to detect. As both the floor plate and notochord are absent in cases in which pathfinding errors are ap parent, and as it is unclear whether some commissural axons have ahnormal trajectories in the absence of the notochord alone, the notochord's role in the guidance of growth cones in the floor plate region remains an open question.

\section{Brain}

The growth cones of specific clusters of brain neurons also follow precise cluster-specific pathways in the early zebrafish brain. Pathfinding within the early scaffold of tracts by the growth cones of one cluster, the nucleus of the posterior commissure (nucPC), located near the forebrain/midbrain border, has recently been analyzed experimentally $[20 \bullet \cdot$. The midbrain contains two bilaterally paired longitudinal tracts, the tract of the postop tic commissure (TPOC) and the medial longitudinal fas ciculus (MLF), connected by the posterior commissure (PC). NucPC growth cones extend ventrally along the $\mathrm{PC}$ to the anterior tegmentum where the PC intersects the TPOC and MLF. At this intersection nucPC growth cones, in principle, could turn anteriorly or posteriorly onto either the TPOC or the MLF, but they virtually always turn posteriorly to fasciculate with the TPOC axons in the dorsolateral tegmentum and follow these axons into the hindbrain (Fig. 2a).

The turning behavior of the nucPC growth cones could occur because they selectively fasciculate with the TPOC axons, respond to longitudinal pathfinding cues indepen-

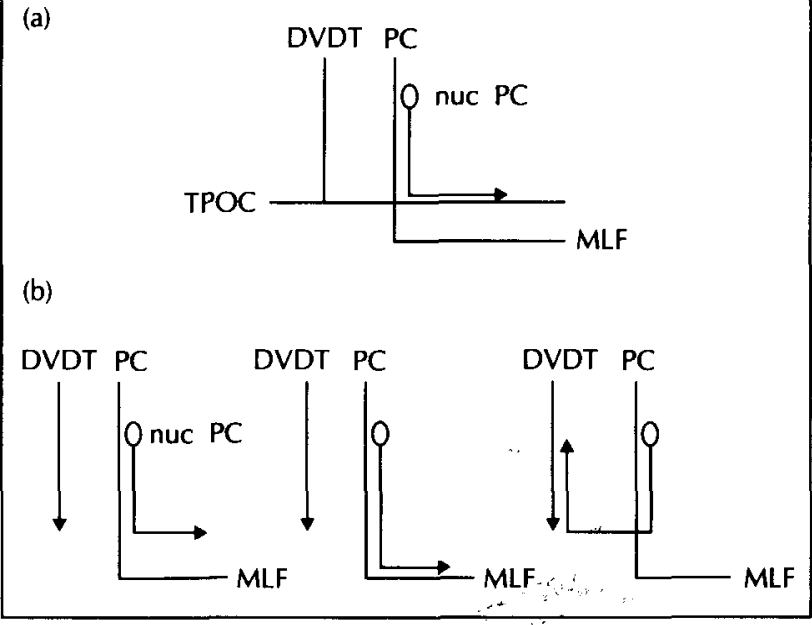

Fig. 2. (a) The pathway taken by the nucleus of the posterior commissure (nucPC) growth cones along the early scaffold of tracts in the brain. Only the portion of the scaffold in the diencephalon and midbrain is depicted. DVDT, dorsoventral diencephalic tract. MLF, medial longitudinal fasciculus. TPOC, tract of the posterior commissure. (b) Three examples of the pathways taken by the nucPC growth cones in the absence of the TPOC. NucPC growth cones follow either normal or aberrant pathways. For more details see text.

dent of the TPOC axons in the dorsolateral tegmentum, or are prevented from entering incorrect pathways. These alternatives have been tested by surgically preventing the TPOC axons, which are projected by a cluster of neurons in the forebrain, from entering the midbrain. Following this manipulation, nucPC growth cones followed either their normal longitudinal pathway in the dorsolateral tegmentum (61\%) or inappropriate pathways (39\%) (Fig. 2b). Growth cones following their normal pathway extended along the endfeet of neuroepithelial cells located in the dorsolateral tegmentum (AB Chitnis et al: Soc Neurosci Abstr 1991, 17:40). Nearly all the growth cones following aberrant pathways followed other tracts, and growth cones from cells within a single nucPC often followed both normal and aberrant pathways. These results support the following conclusions. First, selective fasciculation is important for guiding nucPC growth cones, as in the absence of the TPOC axons a significant proportion of nucPC growth cones make mistakes. Second, inhibition of nucPC growth cones along other tracts is not likely to be a dominant factor during normal pathfinding as nucPC growth cones can follow other tracts in experimental embryos. Third, nucPC growth cones are not obligated to follow each other as they often take divergent pathways in the absence of the TPOC. Fourth, pathfinding cues associated with the dorsolateral tegmentum independent of the TPOC axons, perhaps located along a longitudinal strip of neuroepithelial endfeet, also appear to play an important role in guiding nucPC growth cones as many can follow normal pathways despite the absence of the TPOC axons. Multiple redundant cues therefore appear to act to guide growth cones in the brain as well as in the spinal cord of zebrafish embryos. 


\section{Multiple redundant mechanisms of growth} cone guidance

Multiple redundant cues that guide growth cones at a choice site may involve one or more molecules expressed by more than one cell or group of cells, or a number of molecules on a single cell or group of cells. Such multiple cues may occur in a variety of animals as the elimination of putative pathfinding cues can result in growth cones following both normal and abnormal pathways in several different species. First, in the grasshopper central nervous system the $G$ growth cones turn correctly, but extend much less than normal or fail to make their correct turn, following ablation of the axons which they normally turn onto [21]. Second, blocking the action of the fasciclin 2 adhesion molecule in the grasshopper central nervous system, by application of a specific antibody, caused growth cones that normally express fasciclin 2 to either extend much less along an apparently normal pathway or extend along abnormal pathways [22]. Third, mutations in several unc genes in the nematode lead to an increased number of pathfinding errors made by identified neurons; but the same neurons can also have normal trajectories [23••]. Fourth, genetic suppression of putative guidepost cells in the Drasophila wing does not prevent normal pathfinding by wing neurons, but may increase errors in pathfinding by these cells [24].

The existence of multiple redundant cues is also consistent with a decrease in axonal outgrowth following the elimination of pathfinding cues. In vitro, the growth cones of chick ciliary ganglion cells extend less along axons following the application of antibodies against several adhesion molecules normally found on the axons, but extension was not completely eliminated [25]. In vivo, follower growth cones extend along apparently normal pathways, but the amount they extend decreases after ablation of the appropriate pioneer axons in the embryonic spinal cord of the Japanese medaka fish, and the central nervous system of grasshopper embryos $[7,21]$. Axons are similarly affected following antibody. block of axonal glycoproteins in the grasshopper central nervous system [22].

Multiple cues have been directly demonstrated at the molecular lcvel in two ways. First, the in vitro application of specific antibodies against adhesion molecules, $\boldsymbol{\beta} 1$-integrin or $\mathbf{n}$-cadherin, to chick ciliary neurons grown on glia decreases, but does not prevent, neurite outgrowth. Blocking both molecules simultaneously, however, does prevent neurite outgrowth [26]. Second, Drosophila embryos defective in the fas 1 or abl gene loci, which encode putative adhesion and signal transduction molecules, respectively, exhibit little disruption in axonal outgrowth. Extensive pathfinding errors are observed, however, in double mutants defective in both

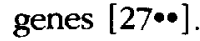

Although some growth cones appear to rely on the simultaneous action of several guidance cues, not all growth cones necessarily require multiple cellular cues. For example, some follower growth cones follow incorrect pathways, or do not extend at all after ablation of other pioneer axons in the grasshopper central nervous system $[28,29]$.

\section{Perspective}

Experimental analyses of pathfinding by growth cones, including those of the zebrafish embryo, clearly demonstrate that growth cones actively select appropriate pathways, and suggest that multiple redundant cues may be opcrative in insuring normal error-free pathfinding by these growth cones. Future investigations should identify the sources of putative redundant cues and help us understand how multiple redundant cues may interact with growth cones. This is especially promising in the simple and well characterized spinal cord of the zebrafish embryo.

\section{References and recommended reading}

Papers of particular interest, published within the annual period of re view, have been highlighted as:

- of special interest

.. of outstanding interest

1. BERNHARDT RR, CHTTNIS AB, LINDAMER L, KUWADA JY: Identification of Spinal Cord Neurons in Embryonic and Larval Zebrafish. J Comp Neurol 1990, 302:607-616.

2. KUWADA JY, BERNHARDT RR, NGUYEN N: Development of Spinal Neurons and Tracts in the Zebrafish Embryo. $J$ Comp Neurol 1990, 302:617-628.

3. Kuwada JY, Bernhardt RR, ChItNis AB: Pathfinding by Iden- tified Growth Cones in the Spinal Cord of Zebrafish Embryos. J Neurosci 1990, 10:1299-1308.

A detailed account of the cell-specific behaviors exhibited by identified growth cones at the floor plate in the spinal cord of the zebrafish embryo. This paper illustrates how the simplicity of this system has allowed investigators to perform highly detailed experimental analyses of growth cone guidance mechanisms.

4. Chitnis AB, Kuwada JY: Axonogenesis in the Brain of Zebrafish Embryos. I Neurasci 1990, 10:1892-1905.

Along with [5*] this paper describes the simple scaffold of tracts and the iclentifiable cell clusters that establish the scaffold in the early ze brafish brain. The report also describes the stereotyped route taken by the nucPC growth cones within the brain scaffold and identifies a potential site where these growth cones might choose among several pathways.

5. WILSON SW, KOSS IS, PARRETT ' $I$, EASTER SJ: The Development - of a Simple Scaffold of Axon Tracts in the Brain of the Embryonic Zebrafish Bracbydanio rerio. Development 1990, 108:121-145.

Along with [ $\left.4^{\bullet}\right]$ this paper describes the simple scaffold of tracts found in the early zebrafish brain.

6. WILSON SW, EASTER SJ: A Pioneering Growth Cone in the Embryonic Zebrafish Brain. Proc Natl Acad Sci USA 1991, 88:2293-2296.

7. KuWAdA JY: Cell Recognition by Neuronal Growth Cones in a Simple Vertebrate Embryo. Science 1986, 233:740-746.

8. GOODMAN CS, Bastiani MJ, DOE CQ, DU LAC S, HELFAND SL, KUWADA JY, THOSMAS JB: Cell Recognition During Neuronal Development. Science 1984, 225:1271-1279.

9. O'Leary DDM, BickNese Ja, DE CARLOS DA, HEFFNER CD, - Koester SE, KutKa lj, Terashima T: Target Selection by 
Cortical Axons: Alternative Mechanisms to Establish Axonal Connections in the Developing Brain. Cold Spring Harb Symp Quant Biol 1990, LV:453-468.

The different ways in which mammalian cortical neurons establish their axon trajectories are discussed in this review. Layer 5 neurons establish their mature trajectories by first projecting growth cones which follow cell-specific pathways within the cortex. Beyond this initial phase, ax onogenesis involves the extension of collateral branches to potential targets followed by selective elimination of a subset of the branches. This also occurs in other organisms including chicks and leeches.

10. Bovalenta P, DODD J: Guidance of Commissural Growth - Cones at the Floor Plate in Embryonic Rat Spinal Cord. Development 1990, 109:435-447.

A clear demonstration that the axons of commissural growth cones turn precisely at the lateral border between the floor plate and the rest of the spinal cord.

11. Yaginuma H, Homma S, Kunzi R, Oppenheim RW: Pathfinding by Growth Cones of Commissural Interneurons in the Chick Embryo Spinal Cord: a Light and Electron Microsopic Study. J Comp Neurol 1991, 304:78-102.

12. ROBERTS A, DAIE N, OTTERSEN OP, STORM-MATHISEN J: Development and Characterization of Commissural Interneurones in the Spinal Cord of Xenopus laevis Embryos Revealed by Antibodies to Glycine. Development 1988, 103:447-461.

13. Dodd J, Morton SB, Karagogeos D, Yamamoto M, Jessell. TM: Spatial Regulation of Axonal Glycoprotein Expression on Subsets of Embryonic Spinal Neurons. Neuron 1988, 1:105-111.

14. Tessier-Lavigne M, Placzek M, Lumsden AGS, DOdD J, JESSELI. TM: Chemotropic Guidance of Developing Axons in the Mammalian Central Nervous System. Nature 1989 336: $775-778$

15. YaGINUMA H, OPPENHEIM RW: An Experimental Analysis of In - Vivo Guidance Cues Used by Axons of Spinal Interneurons in the Chick Embryo: Evidence for Chemotropism and Related Guidance Mechanisms. $J$ Neurasci 1991, 11:2598-2613. Spinal cord grafts rotated relative to the host spinal cord demonstrate that commissural axons near the graft/host boundary are attracted to the nearest set of floor plate cells.

16. HatTA K, Kimmel CB, HO RK, WalKer C: The Cyclops Muta-. tion Blocks Specification of the Floor Plate of the Zebrafish Central Nervous System. Nature 1991, 350:339-341.

This important paper illustrates the power of the genetic approach for the analysis of the development of zebrafish embryos by characterizing the cyc-1(b16) mutation in zebrafish embryos. It demonstrates that in the spinal cord the mutation deletes the midline foor plate cells. The elegant analysis of mosaic embryos demonstrates that the mutation acts cell autonomously and that floor plate cells can be induced by other floor plate cells.

17. Kimmel CB, POWEll SL, METCAIFE WK: Brain Neurons which Project to the Spinal Cord in Young Larvae of the Zebrafish. $J$ Comp Neurol 1982, 205:112-127.

18. BOVOLENTA P, DODD J: Perturbation of Neuronal Differentiation and Axon Guidance in the Spinal Cord of Mouse Embryos Lacking a Floor Plate: Analysis of Danforth's Short Tail Mutation. Development 1991, 113:625-639.

19. Clarke JDW, HOLder N, SOFFe SR, StORM-MATHISEN J: Neuroanatomical and Functional Analysis of Neural Tube Formation in Notochordless Xenopus Embryos; Laterality of the Ventral Spinal Cord is Lost. Development 1991, 112:499-516.
20. CHITNIS AB, KUWADA JY: Elimination of a Brain Tract In-. creases Errors in Pathfinding by Follower Growth Cones in the Zebrafish Embryo. Neuron 1991, 7:277-285.

This study takes advantage of the detailed description of the behavior of a specific set of growth cones at a potential choice site in the zebrafish brain. Elimination of the axons which these growth cones normally turn onto at this site causes the growth cones to follow either aberrant or correct pathways. This study illustrates the usefulness of the zebrafish brain for andlysis of growth cone guidance in the vertebrate brain.

21. Raper Ja, Bastiani MJ, Goodman CS: Pathfinding by Neuronal Growth Cones in Grasshopper Embryos. IV. The Fffects of Ablating the $A$ and $P$ Axons Upon the Behavior of the $G$ Growth Cone. J Neurosci 1984, 4:2329-2345.

22. HARRELSON AL, GOODMAN CS: Growth Cone Guidance in Insects: Fasciclin II is a Member of the Immunoglobulin Superfamily. Science $1988,242: 700-708$

23. HedGeCoCK EM, CulotT JG, Hall DH: The Unc-5, Unc-6, -. and Unc- 40 Genes Guide Circumferential Migrations of Pioneer Axons and Mesodermal Cells on the Epidermis in $C$. elegans. Neuron 1990 4:61-85. ...

This paper describes in detail the effects of mutations in three genes on navigation by identified growth cones and migration by mesodermal cells in the nematode. It clearly demonstrates with a multi-allele analysis that mutations in these genes can cause pioneer growth concs to follow variable pathways as well as their normal ones.

24. SCHIJIGER M, PAIKA J: Genetic Suppression of Putative Guidepost Cells: Effect on Establishment of Nerve Pathways in Drosopbila Wings. Dev Biol 1985, 108:399-410.

25. CHANG S, RATHJEN FG, RAPER JA: Extension of Neurites on Axons is Impaired by Antibodies Against Specific Neural Cell Surface Glycoproteins. $J$ Cell Biol 1987, 104:355-362.

26. TOMASEII KJ, Neugebauer KM, BIXBY JL, LILIEN J, Reichardt $L F$ : N-Cadherin and Integrins: Two Receptor Systems that Mediate Neuronal Process Outgrowth on Astrocyte Surfaces. Neuron 1988, 1:33-43.

27. EIKINS T, ZINN K, MCALLISTER L, HOFFMANN FM, GOODMAN -. CS: Genetic Analysis of a Drosopbila Neural Cell Adhesion Molecule: Interaction of Fasciclin I and Abelson Tyrosine Kinase Mutations. Cell 1990, 60:565-575.

This experiment uses the advanced molecular genetics of Drasophila to clarify the roles of putative pathfinding genes. Most importantly it demonstrates that the growth cones of neurons, e.g. RP1, which express both the fasciclin I ( fas I) adhesion molecule and the Abelson tyrosine kinase $(a b l)$ protein show no gross defects in mutants defec tive in one of the genes, but clearly follow aberrant pathways in double mutants. This vividly demonstrates that the fas I and $x b l$ genes interact. Double mutants homozygous for the fas $I$ and numerous other genes, including other adhesion molecule genes, however, show little phenotype. Interestingly $7 \%$ of RP1 neurons still follow normal pathways even in the fas I abl double mutants.

28. BASTIANI MJ, DU LAC S, GOODMAN CS: Guidance of Neuronal Growth Cones in the Grasshopper Embryo. I. Recognition of a Specific Axonal Pathway by the pCC Neuron. $J$ Neurosci 1986, 6:3518-3531

29. DU LAC S, BASTIANI MJ, GOODMAN CS: Guidance of Neuronal Growth Cones in the Grasshopper Embryo. II. Recognition of a Specific Axonal Pathway by the aCC Neuron. $J$ Neurasci 1986, 6:3532-3541.

JY Kuwada, Department of Biology, Natural Science Building, University of Michigan, Ann Arbor, Michigan 48109-1048, USA. 